MATEC Web of Conferences 24,05003 (2015)

DOI: $10.1051 /$ matecconf/ 20152405003

(C) Owned by the authors, published by EDP Sciences, 2015

\title{
One-year dynamic monitoring of a masonry tower
}

\author{
Marco Guidobaldi ${ }^{1}$, Carmelo Gentile ${ }^{1, a}$ and Antonella Saisi ${ }^{1}$ \\ ${ }^{1}$ Politecnico di Milano, Department of Architecture, Built environment and Construction engineering, Milan, Italy
}

\begin{abstract}
The paper presents some results of the continuous dynamic monitoring program carried out on the tallest historic tower in Mantua, Italy. This project follows an extensive diagnostic investigation aimed at assessing the structural condition of the tower after the Italian earthquakes of May 2012. A simple dynamic monitoring system was permanently installed in the upper part of the building and automatic modal identification was performed. The results allow to evaluate the effects of changing temperature on automatically identified natural frequencies, to verify the practical feasibility of damage detection methods based on natural frequencies shifts and provide clear evidence of the possible key role of continuous dynamic monitoring in the preventive conservation of historic towers.
\end{abstract}

\section{Introduction}

Ancient masonry towers represent a significant part of the existing Cultural Heritage buildings, as these historic structures were built with different characteristics and functions: bell towers (especially meaningful in Italy, considering the high number of historic churches), lookout or defensive towers, chimneys and minarets. In addition to the general issues characterizing the preservation of historic buildings (i.e. ageing of materials, presence of cracks and damage, effects of successive adding or modifications, lack of connection between structural elements), masonry towers are usually slender and subjected to significant dead loads. Such important aspects - as well as the presence of adjacent buildings and large openings at belfries - may often lead to high sensitivity or vulnerability to dynamic actions, such as traffic-induced micro-tremors, swinging of bells, wind and earthquakes. Hence, the preservation and preventive conservation of historic towers represent a major challenge.

Since masonry towers are generally sensitive also to the ambient excitation, dynamic tests in operational conditions were more frequently adopted in recent studies [1-5] and should be considered a fundamental step in the diagnostic process of these buildings. Furthermore, the cantilever-like behaviour of towers suggests the use of simple dynamic monitoring systems, consisting of few accelerometers installed in the upper part of the building, with preventive conservation and/or Structural Health Monitoring (SHM) purposes [3, 5].

Therefore, a good process of preventive conservation of historic masonry towers should consist of:

1. data collection of the structure, including historic and documentary research, on-site and topographic survey, visual inspection, non-destructive and minor- destructive tests of materials on site, dynamic tests in operational conditions (with a number of sensors sufficient to completely describe the dynamic characteristics of the building) and development of experimentally validated FE models;

2. health monitoring, performed by installing a limited number of sensors in the structure (at least 3 accelerometers and 1 temperature sensor). Data should be collected continuously or periodically and automated Operational Modal Analysis (OMA) should be carried out to investigate the environmental effects and the occurrence of structural performance anomalies.

After the SHM revealed a possible deterioration of the structural condition, local assessment of damage should be carried out through detailed visual inspection and complementary non-destructive tests.

The paper exemplifies the application of SHM based on OMA within the preservation process of the tallest historic tower in Mantua, Italy: the Gabbia tower [6-7].

After the seismic sequence occurred in Italy in May 2012, an extensive research program was carried out to assess the structural condition of the tower [7]. The postearthquake investigation (including direct survey, historic and documentary research, testing of materials and ambient vibration tests) highlighted the poor state of preservation of the upper part of the structure and suggested the installation of a dynamic monitoring system, as a part of the health monitoring process aimed at preserving the historic tower.

The instrumentation installed inside the tower consists of a 4-channel data acquisition board, with 3 piezoelectric accelerometers and 1 temperature sensor. The main objectives of the continuous dynamic monitoring are: (a) evaluating the dynamic response of the structure to the expected sequence of far-field earthquakes; (b) evaluating

\footnotetext{
${ }^{\mathrm{a}}$ Corresponding author: carmelo.gentile@polimi.it
} 
and modelling the effects of environmental factors on the natural frequencies of the tower; (c) detecting any possible anomaly or change in the structural behaviour.

After a brief description of the tower and the postearthquake survey, the paper presents the results of the first 15 months of monitoring, highlighting the effect of temperature on the automatically identified natural frequencies and the practical feasibility of damage detection methods based on natural frequency shifts and novelty indices.

\section{SHM methodology}

After the preliminary investigation [7] needed to understand the actual structural condition of the tower, a vibration-based SHM strategy was herein adopted, consisting of the following phases:

\section{(1) Preliminary ambient vibration tests}

Preliminary ambient vibration tests (AVTs) should be characterized by the use of an appropriate number of sensors (e.g. accelerometers, temperature sensors, etc.) in order to: (i) identify the dynamic characteristics of the tower, to be used as reference for the subsequent long-term monitoring; (ii) highlight the most meaningful positions to be permanently instrumented and the environmental factors worth measuring;

(2) Continuous dynamic monitoring and automated modal identification

In principle, the cantilever-like behaviour of towers allows an effective health monitoring by installing a limited number of sensors in the upper part of the building. At least three accelerometers are needed to correctly capture the dynamic characteristics of the structure at the instrumented level, along with one temperature sensor to measure the temperature variations.

In the present paper, the modal parameters of the tower were extracted from the measured acceleration data using an automated procedure [5] based on the covariance-driven Stochastic Subspace Identification (SSI-Cov) algorithm [8-9].

In the SSI-Cov algorithm, the covariance matrices of the $m$ outputs are evaluated, for positive time lags varying from $1 \Delta t$ to $(2 i-1) \Delta t$, to fill a $(m i \times m i)$ block Toeplitz matrix, that is decomposed to obtain stochastic subspace models (i.e. matrices $\boldsymbol{A}, \boldsymbol{C}$ ) of increasing order $n$. In the automated procedure [5], each resulting stabilization diagram (where the modes obtained with increasing model order are represented together) was firstly "cleaned" from certainly spurious modes by checking the non-physical characteristics of each point (i.e. mode) of the stabilization diagram through mode shape complexity as well as stability and range of damping ratios [10]. Similar modes in the cleaned stabilization diagram are grouped together and the modal parameters are estimated, based on the sensitivity of frequencies and mode shapes to an increasing model order. Finally, the identified frequencies and modal deflections are compared to the reference results estimated in the preliminary tests.

In the present application, the time lag parameter $i$ was set equal to 70 and the data was fitted using stochastic subspace models of order varying between 30 and 120 ;

(3) Regression analysis to highlight the environmental effects on natural frequencies

As measuring the dynamic response using few accelerometers provides information mainly on the natural frequencies, these parameters have to be necessarily used as features sensitive to damage. However, the natural frequencies of masonry towers are also affected by factors other than damage, such as temperature $[3,5,11-12]$. Hence, the correlation between natural frequencies and temperature needs to be investigated for early detection of damage: once the normal response of a structural system to changes in its environmental conditions has been explored and can be filtered out to normalize the response data, any further changes in the sensitive features should rely to changes of the structural condition.

\subsection{Temperature effects on natural frequencies}

In order to characterize the effects of temperature on the natural frequencies, a dynamic regression model has been built for each identified modal frequency. Dynamic regression models [13] assume that the value $y_{\mathrm{k}}$ of the dependent variable (i.e. natural frequency) at time instant $k$ is influenced by the values of the model input (i.e. temperature) at current time $k$ as well as at $(p-l)$ previous time instants:

$$
y_{\mathrm{k}}=b_{1} x_{\mathrm{k}}+b_{2} x_{\mathrm{k}-1}+\ldots+b_{\mathrm{p}} x_{\mathrm{k}-(\mathrm{p}-1)}+\varepsilon_{\mathrm{k}}
$$

The linear model (1) can be expressed in matrix form as:

$$
\boldsymbol{y}=\boldsymbol{X} \boldsymbol{b}+\varepsilon
$$

where $\boldsymbol{y} \in \mathfrak{R}^{n}$ is the vector containing $n$ observations $y_{\mathrm{k}}$ of the dependent variable, $\boldsymbol{b} \in \mathfrak{R}^{p}$ is the vector formed by the $p$ parameters weighting the contribution of the input, $\boldsymbol{\varepsilon} \in \mathfrak{R}^{n}$ is the vector of the random errors and $\boldsymbol{X} \in \mathfrak{R}^{n \times p}$ is the matrix appropriately gathering (see eq. (1)) the values of the input.

It is worth mentioning that: (a) in the framework of a dynamic monitoring project, different input candidates might be considered in the dynamic regression model (1)(2), such as temperature, humidity, wind speed, traffic loads on bridges, etc. (see e.g. [14-15]); (b) the model (1)-(2) can be further generalized so that predictions are computed considering also previous values of the dependent variables [16].

In order to properly describe the influence of changing environmental conditions, the data used to estimate the parameters of the regression models should be collected over a significant period of time, denoted as training/reference period and including a statistically representative sample of temperature conditions.

After a regression model (2) has been established for each natural frequency, the occurrence of anomalies not 
observed during the training period might be detected by using:

a) The residual error vectors $\boldsymbol{\varepsilon}_{\mathrm{j}}=\boldsymbol{y}_{\mathrm{j}}-\boldsymbol{X}_{\mathrm{j}} \boldsymbol{b}_{\mathrm{j}}(j=1,2, \ldots$, $M$ where $M$ is the number of automatically identified modal frequencies);

b) The cleaned observation vectors $\boldsymbol{y}_{\mathrm{j}}{ }^{*}=\mathbf{1} \mu_{\mathrm{j}}+\varepsilon_{\mathrm{j}}(j=1$, $2, \ldots, M$ where $\mathbf{1}$ is the unit vector and $\mu_{\mathrm{j}}$ is the mean value of the original $j$-th frequency data in the reference period);

c) The prediction error vectors;

as all these quantities conceivably contain frequency data without the effect of changing environment.

Alternatively, the occurrence of structural anomalies can be detected by using the statistical tools defined as "control charts". A control chart typically consists of a plot where the variation in time of a feature is represented along with a user-defined variation limit. The designated control limit, computed from the experimental samples collected when the process is supposed to be in control (training period), allows to check if the fluctuations of the monitored feature are associated to the normal response. Any observation laying outside the control limit has to be considered the result of unusual sources of variability (e.g. the occurrence of damage).

In this paper, the multivariate control chart designated as Shewhart $T$ [17] has been applied. The characteristic plotted in the Shewhart $T$ chart is the $T^{2}$-statistic.

Let us denote $\boldsymbol{z} \in \mathfrak{R}^{M}$ the observation vector collecting the residual errors of the $M$ available natural frequencies at a certain time instant. Future observations are checked by using subgroups with $r$ observations of the vector $z$, and the $T^{2}$-statistic is calculated as:

$$
T^{2}=r[\overline{\boldsymbol{z}}-\hat{\boldsymbol{z}}]^{\mathrm{T}} \boldsymbol{S}^{-1}[\overline{\boldsymbol{z}}-\hat{\boldsymbol{z}}]
$$

where $\overline{\boldsymbol{z}}$ is the mean of the subgroup of the new $r$ observations, $\hat{\boldsymbol{z}}$ and $\boldsymbol{S}$ are the process average and covariance matrix, respectively, computed during the reference period.

(a)

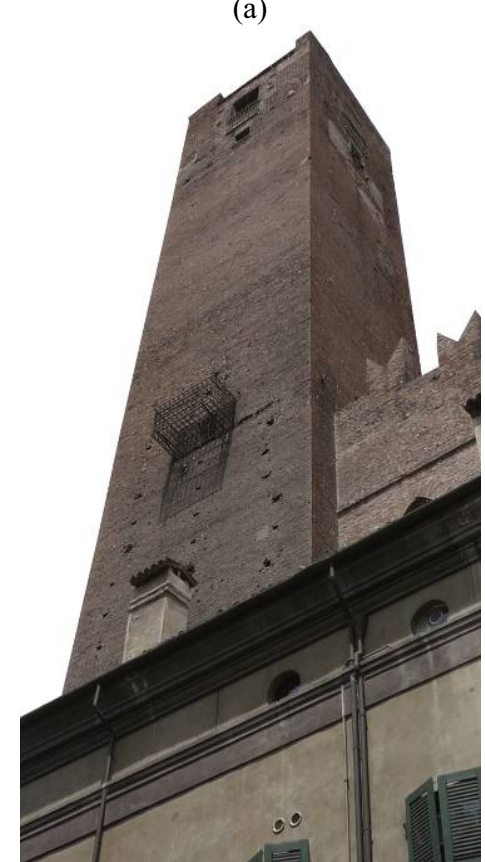

(b)

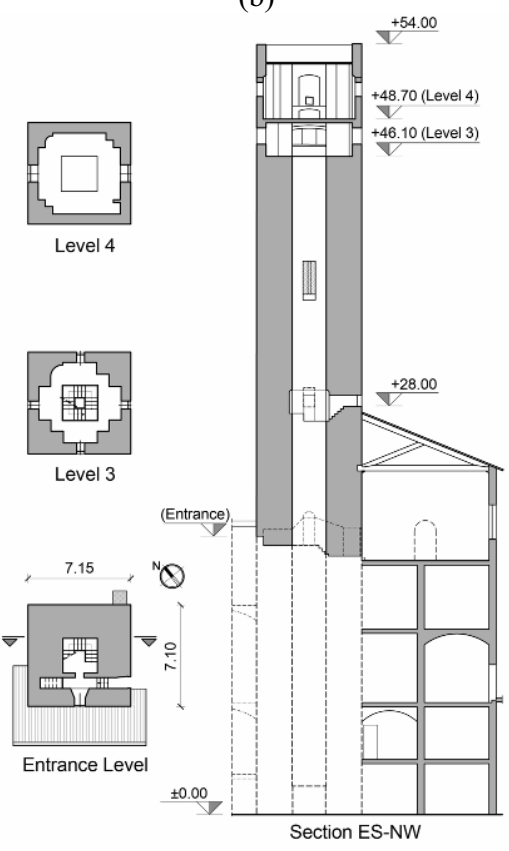

The Lower Control Limit (LCL) is set equal to zero whereas the Upper Control Limit (UCL) is defined as:

$$
\mathrm{UCL}=[M(s+1)(r-1) /(s r-s-M+1)] F(\gamma)
$$

where $s$ is the number of subgroups collected during the reference period (used to estimate the process average $\hat{z}$ and covariance matrix $\boldsymbol{S}$ ) and $F(\gamma)$ denotes the $\gamma$ percentage point of the $F$ distribution with $M$ and $s r-s-M+1$ degrees of freedom.

\section{Description of the Gabbia tower and on-site tests}

The Gabbia tower (Fig. 1) [6], about $54.0 \mathrm{~m}$ high and with almost square plan, is the tallest tower in Mantua and is named after the hanged dock built in the XVI century on the S-W front (Fig. 1a) and originally used as open-air jail. The tower was part of the defensive system of the Bonacolsi family (the Lords of Mantua during the XIII century) and some recent research dates the end of construction to 1227 .

The tower is nowadays part of an important palace (Figs. 1a and 1b) whose load bearing walls seem to be not effectively linked but just drawn to the tower's masonry walls, whereas the tower directly supports several floors and vaults. The structure is built in solid brick masonry and the load-bearing walls are about $2.4 \mathrm{~m}$ thick except for the upper levels, where the section decreases to about $0.7 \mathrm{~m}$ and a two level lodge is hosted (Fig. 1b). Over the past centuries, the lodge was used for military and communication purposes and was reachable through a wooden staircase. The staircase has not been practicable since the 1990s due to lack of maintenance and the access to the inside of the tower was re-established only in October 2012, when provisional scaffoldings were installed in order to allow visual inspection and geometric survey of the inner load-bearing walls.

No extensive information is available on the tower

Figure 1. (a) S-W view of the Gabbia tower; (b) Sections (dimensions in m); (c) Details of the upper region and building phases 
a)

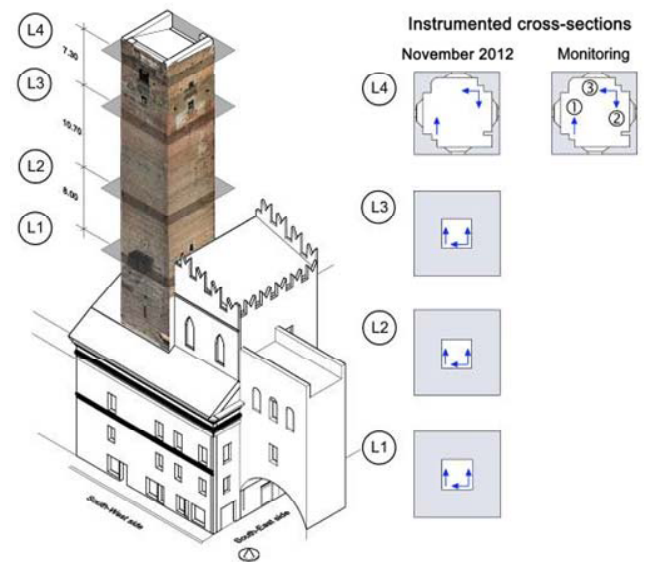

b)

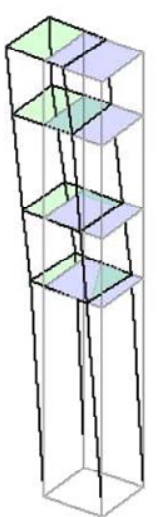

$f=0.918 \mathrm{~Hz} \quad f=0.986 \mathrm{~Hz} \quad f=3.887 \mathrm{~Hz}$

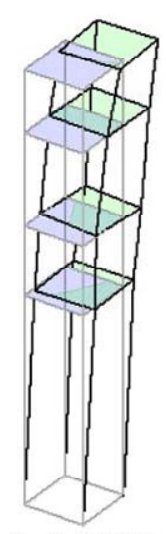

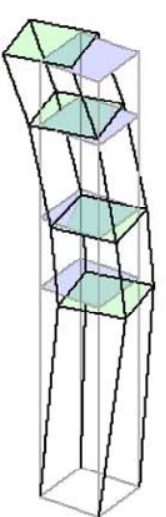
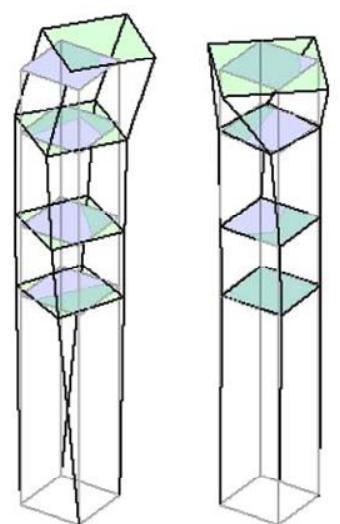

$f=4.648 \mathrm{~Hz} \quad f=9.893 \mathrm{~Hz}$

Figure 2. (a) Sensors layout (dimensions in $\mathrm{m}$ ) adopted in the preliminary AVT and in the continuous dynamic monitoring of the Gabbia tower; (b) Vibration modes identified in the preliminary AVT (SSI-Data, 27/11/2012)

past interventions but the passing-through discontinuities detected at the upper levels by the stratigraphic survey can be conceivably referred to evolution phases of the building. Indeed, the results of the survey allowed to recognize at least six main building phases, partially reported in Fig. 1c. Moreover, a clear change of the brick surface workmanship observed at about $8.0 \mathrm{~m}$ from the top suggests a first addition.

After the Italian seismic sequence of May 2012, accurate on-site survey of all outer fronts of the Gabbia tower was performed using a movable platform and visual inspection [7] highlighted two different structural conditions for the tower: (a) in the main part of the building, until about $46.0 \mathrm{~m}$ from the ground level, no evident structural damage was observed and the materials appeared mainly affected by superficial decay; (b) the upper portion of the tower (i.e. the top $8.0 \mathrm{~m}$ ) is characterized by extensive masonry decay, traces of past structures on all fronts and merlon-shaped discontinuities (Fig. 1c). The poor state of preservation and the vulnerability of the top levels of the structure were confirmed by the results of pulse sonic tests and flat-jack tests [7].

Dynamic tests in operational conditions were performed on 27/11/2012. The structural response was measured by high sensitivity accelerometers (WR model $731 \mathrm{~A}, 10 \mathrm{~V} / \mathrm{g}$ sensitivity and $\pm 0.50 \mathrm{~g}$ peak) in 12 points, belonging to 4 pre-selected cross-sections (Fig. 2a) and adopting a sampling frequency of $200 \mathrm{~Hz}$. The tests were carried out after the installation of a metallic scaffolding and a wooden roof inside the tower and all the accelerometers were installed on the inside of the walls.

After filtering and decimating the recorded data, the extraction of the modal parameters was carried out by using the data-driven SSI (SSI-Data) algorithm, available in the commercial software ARTeMIS [18], and considering datasets of $3600 \mathrm{~s}$. Notwithstanding the very low level of ambient excitation, 5 vibration modes were identified within the frequency range $0-10 \mathrm{~Hz}$ (Fig. 2b):

1. Two closely spaced modes were identified around 1.0

$\mathrm{Hz}$. These modes are dominant bending (modes $\mathrm{B}_{1^{-}}$

$\mathrm{B}_{2}$ ) and involve flexure in the two main planes of the tower, respectively;

2. The third mode (mode $\mathrm{B}_{3}$ ) involves dominant bending in the N-E/S-W plane with slight components also in the orthogonal N-W/S-E plane;

3. The fourth mode (mode $T_{1}$ ) involves dominant torsion of the tower until the height of $46.0 \mathrm{~m}$ and coupled torsion-bending of the upper level. The local behaviour of the top region of the tower, which alters what would otherwise be classified as a pure torsion mode, can be conceivably associated to the effect exerted by the wooden roof, directly resting on the most vulnerable region of the tower;

4. One local mode $\mathrm{L}_{1}$ was identified at $9.89 \mathrm{~Hz}$ and involved torsion of the upper part of the tower. The presence of a local mode provides further evidence of the structural effect of the change in the masonry quality and morphology (including un-toothed openings, infillings and discontinuities) observed in the upper part of the tower during the visual inspection.

It is to be further noticed that the wooden roof, although very light, is slightly inclined in the N-E/S-W direction (as schematically shown in Fig. 2a) and is directly supported by the weakest structural part of the building. It is worth underlining that the roof slope, the redundant connection with the masonry walls and the thermal effects might induce not negligible thrusts on the structural walls, already very vulnerable because of the extensive decay, the presence of several discontinuities and the lack of connection of the different adding.

\section{Dynamic monitoring and results}

Few weeks after the execution of the preliminary AVT, a continuous dynamic monitoring system was installed in the tower. The system is composed by: (a) one 4-channels data acquisition system (24-bit resolution and $102 \mathrm{~dB}$ dynamic range); (b) 3 piezoelectric accelerometers (WR model $731 \mathrm{~A}, 10 \mathrm{~V} / \mathrm{g}$ sensitivity and $\pm 0.50 \mathrm{~g}$ peak), mounted on the cross-section at the crowning level of the tower (Fig. 2a); (c) one temperature sensor, installed on the S-W front and measuring the outdoor temperature; (d) one industrial PC on site, for system management and data storage. A binary file, containing 3 acceleration time series and the temperature data, is created every hour, stored in the local PC and transmitted to Politecnico di Milano for being processed. 
(a)

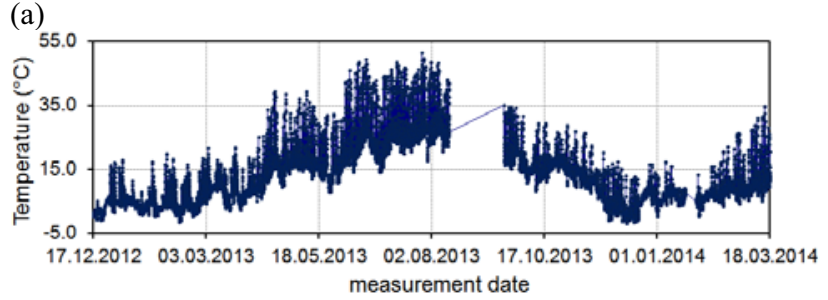

(b)

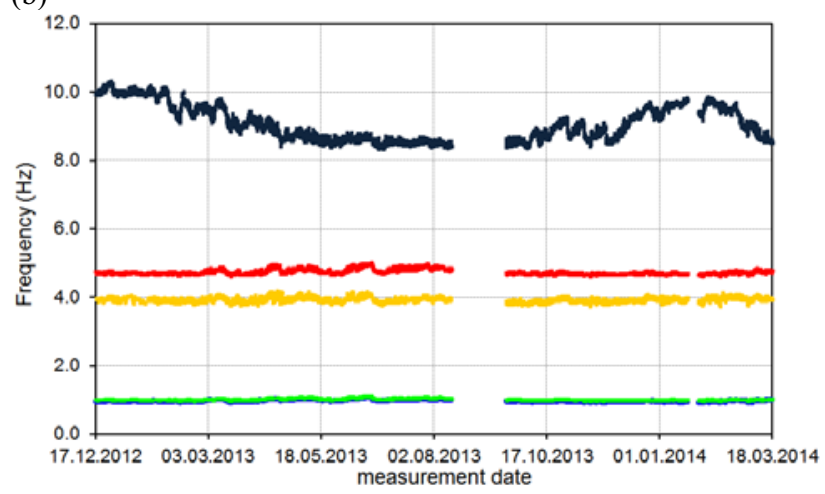

Figure 3. Time evolution of temperature (a) and identified frequencies (b), from $17 / 12 / 2012$ to $17 / 03 / 2014$

The continuous dynamic monitoring system has been active since late December 2012. The data files received from the monitoring system are managed by a LabVIEW toolkit automatically performing the following tasks [19]: (a) creation of a database with the original data (in compact format) for later developments; (b) preliminary pre-processing (i.e. de-trending, automatic recognition and extraction of possible seismic events, creation of 1 dataset per hour); (c) statistical analysis of data, including the evaluation of averaged acceleration amplitudes and temperature trends; (d) low-pass filtering and decimation of each dataset; (e) creation of a second database, with essential data records, to be used in the modal identification phase.

This section summarizes the main results of the continuous monitoring for a period of about 15 months, from $17 / 12 / 2012$ to $17 / 03 / 2013$. During this time interval, 10945 1-h datasets were collected and automated modal identification was performed. It is worth mentioning that between January and June 2013, the monitoring system acquired the tower's response to various far-field earthquakes. The last event, occurred on 21/06/2013 in the Garfagnana region, was the strongest of the recorded seismic episodes and induced significant effects on the Gabbia tower.

Fig. 3 reports the time evolution of the outdoor temperature (S-W front, Fig. 3a) and of the automatically identified modal frequencies (Fig. 3b).

The temperature tracking reveals large fluctuations, between $-2^{\circ} \mathrm{C}$ and $45^{\circ} \mathrm{C}$, with significant daily variations on sunny days.

A closer inspection of Fig. 3b highlights that the natural frequencies of global modes $\left(B_{1}-B_{3}\right.$ and $T_{1}$, Fig. $2 \mathrm{~b})$ vary accordingly with the outdoor temperature. This correlation can be better investigated by plotting each modal frequency with respect to the recorded temperature. Figs. 4a-c show the results obtained for modes $\mathrm{B}_{1}, \mathrm{~B}_{2}$ and $\mathrm{T}_{1}$ along with the best fit lines and the coefficient of determination $R^{2}$ [20]: the three plots refer to the time period from $17 / 12 / 2012$ to $20 / 06 / 2013$ and confirm that the frequency of global modes tends to increase with increased temperature. This behaviour, observed also in past studies of masonry towers $[3,5,11]$ can be explained by the closure of superficial cracks, minor masonry discontinuities or mortar gaps induced by the thermal expansion of materials. Hence, the temporary "compacting" of the materials induces a temporary increase of stiffness and modal frequencies.

It should be noted that no results have been presented for mode $\mathrm{B}_{3}$ on the correlation between natural frequency and temperature. Indeed, the modal identification process for this mode revealed some unexpected difficulties: the presence of spurious harmonics close to the investigated natural frequency (conceivably produced by some active machineries present in the building) often undermines the quality and reliability of the frequency estimates of the mode.

The frequency-temperature relationships obtained after the Garfagnana earthquake (21/06/2013) are shown in Figs. 4d-f. The comparison with the results referred to the first six months of monitoring (Figs. 4a-c) reveals significant differences: the regression lines of all modes exhibit remarkable variations after the earthquake, with the temperature range being almost unchanged. This trend is also confirmed by the general decrease of the statistics of the natural frequencies (mean values, standard deviations, extreme values) summarized in Table 1 and suggests the occurrence of structural changes. The effects induced by the earthquake of 21/06/2013 will be further discussed in section 5 .

The inspection of Table 1 also reveals that the standard deviations are generally larger than $0.03 \mathrm{~Hz}$ for all modes and high values are especially observed for the local mode. Furthermore, Fig. $3 \mathrm{~b}$ highlights that frequency evolution of mode $\mathrm{L}_{1}$ looks very different from the others and characterized by significant frequency variations (approximately in the range 10.33-8.33 Hz).

A thorough examination of the frequency tracking allows to recognize three different time windows: in a first phase (from 17/12/2012 to 14/08/2013) the frequency clearly decreases in time, from an initial value of about $10.0 \mathrm{~Hz}$ to a final value of about $8.5 \mathrm{~Hz}$; after the Summer (from 19/09/2013 to 19/01/2014) the natural frequency increases again, even if the new maximum values do not reach those identified one year before in similar temperature conditions; finally, the modal frequency decreases again from 02/02/2014 to the end of the considered 15 months.

Considering the first time period, 3 drops can be detected: (a) 03/02/2013-04/02/2013; (b) 14/03/2013$15 / 03 / 2013$; (c) $13 / 04 / 2013-15 / 04 / 2013$. These drops divide the analysed interval in 4 parts, easily observable by plotting the modal frequency versus the measured outdoor temperature, as shown in Fig. 5. This figure highlights that the clouds of temperature-frequency points, corresponding to each of the 4 different periods, are characterized by similar slope of the best fit line, whereas the average frequency value significantly decreases. A similar trend can be observed for the subsequent time periods, with the frequency increasing and decreasing, respectively. 
(a)

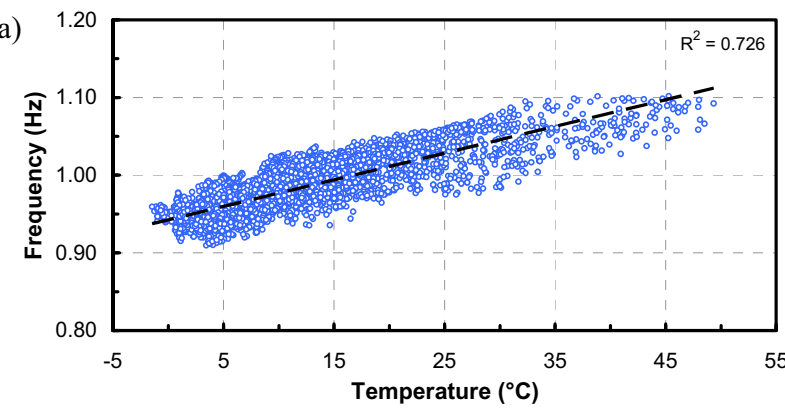

(b)

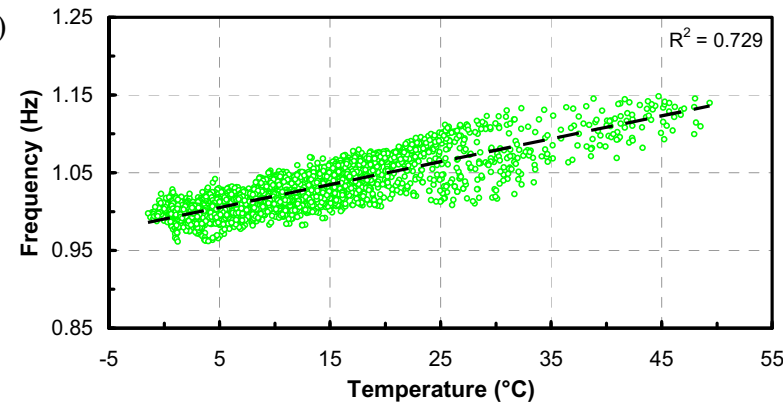

(c)

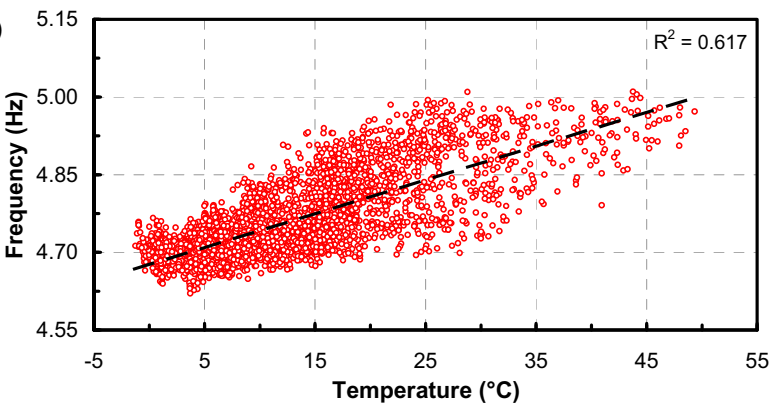

(d)

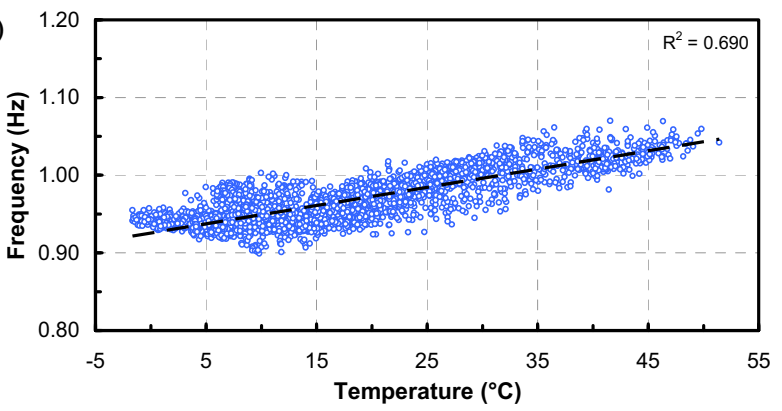

(e)

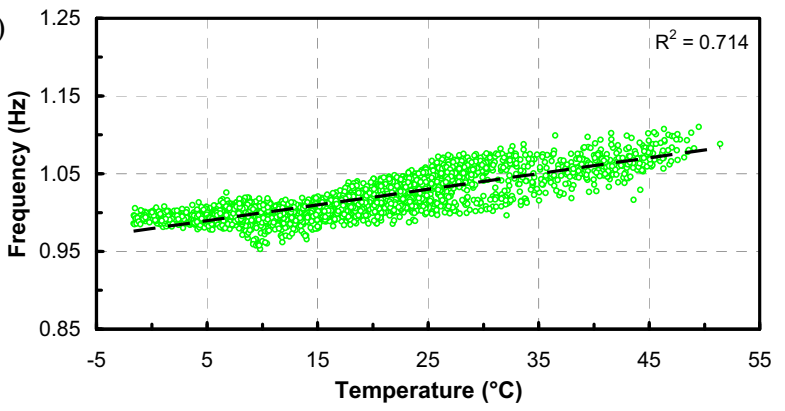

(f)

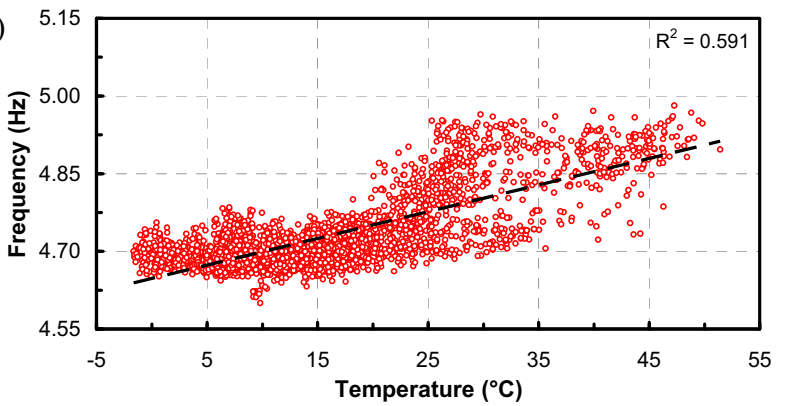

Figure 4. Natural frequency of modes $B_{1}, B_{2}$ and $T_{1}$ plotted with respect to the outdoor temperature: (a)-(c) from 17/12/2012 to 20/06/2013; (d)-(f) after 21/06/2013

Table 1. Statistics of the identified natural frequencies before and after the seismic event of 21/06/2013.

\begin{tabular}{ccccccccc}
\hline \multirow{2}{*}{ Mode } & \multicolumn{2}{c}{$f_{\text {ave }}(\mathrm{Hz})$} & \multicolumn{2}{c}{$\sigma_{f}(\mathrm{~Hz})$} & \multicolumn{2}{c}{$f_{\min }(\mathrm{Hz})$} & \multicolumn{2}{c}{$f_{\max }(\mathrm{Hz})$} \\
\cline { 2 - 8 } & Before & After & Before & After & Before & After & Before & After \\
\hline \hline $\mathrm{B}_{1}$ & 0.985 & 0.968 & 0.038 & 0.031 & 0.910 & 0.897 & 1.102 & 1.070 \\
$\mathrm{~B}_{2}$ & 1.024 & 1.012 & 0.032 & 0.025 & 0.961 & 0.953 & 1.148 & 1.110 \\
$\mathrm{~B}_{3}$ & 3.941 & 3.929 & 0.075 & 0.063 & 3.758 & 3.742 & 4.194 & 4.137 \\
$\mathrm{~T}_{1}$ & 4.754 & 4.727 & 0.077 & 0.066 & 4.621 & 4.600 & 5.010 & 4.982 \\
$\mathrm{~L}_{1}$ & 9.222 & 8.937 & 0.554 & 0.433 & 8.385 & 8.332 & 10.327 & 9.862 \\
\hline
\end{tabular}

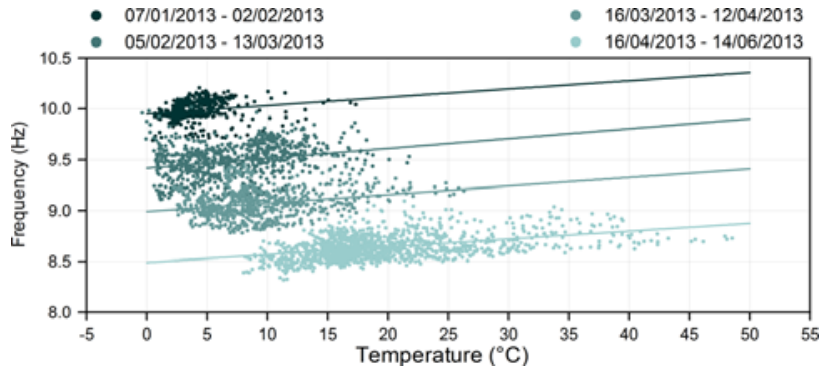

Figure 5. Natural frequency of local mode $L_{1}$ plotted with respect to the outdoor temperature in the period between 07/01/2013 and 14/06/2013

The observed behaviour suggests the progress of a possible damage mechanism, conceivably related to the effect exerted by the wooden roof with increased temperature, and confirms the poor structural condition and the high seismic vulnerability of the upper part of the tower. This assumption seems to be confirmed by the frequency loss of the local mode detected after one year of monitoring, with the natural frequency unable to reach the maximum values identified one year before in a similar temperature range (Fig. 3b).

\section{Temperature and damage effects}

As pointed out in section 2, the effects of temperature on the natural frequency of each global mode have been described by means of a dynamic regression model, with the outdoor temperature measured on the S-W front as its only input. Based on the classic Loss Function (LF) and 
the Final Prediction Error (FPE) criteria [16], regression models depending on the current and 13 previous hourlyaveraged values of the external temperature have been established.

As the main goal was to mitigate the environmental effects, in order to detect possible structural changes induced by the earthquake of $21 / 06 / 2013$, the models were trained over the 6 months preceding that seismic event (where the process is known to be in control). The comparison between the identified natural frequencies and the corresponding predictions (Fig. 6) highlights a very good agreement, providing a sound validation of the adopted dynamic regression models.

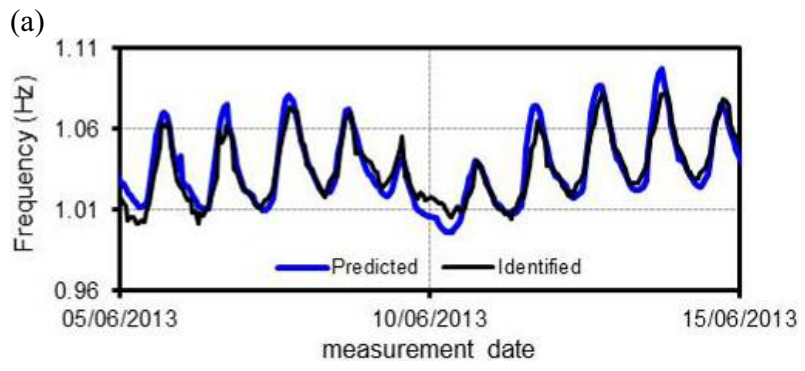

(b)

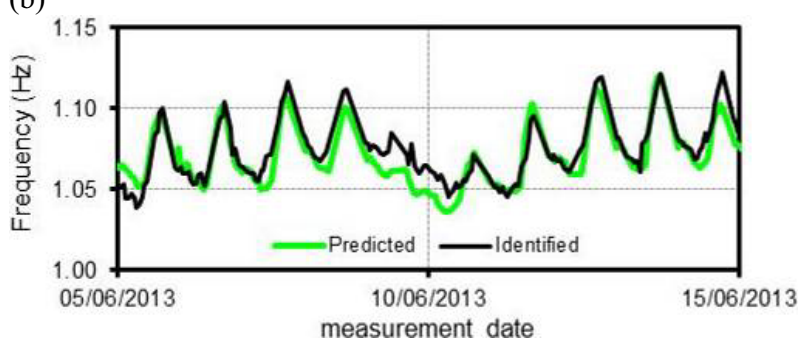

(c)

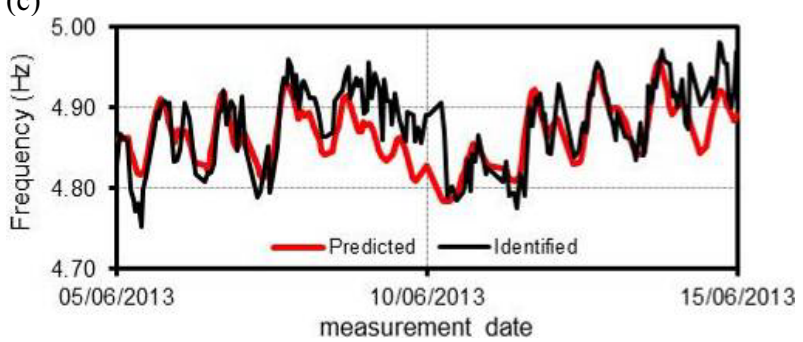

Figure 6. Typical prediction of natural frequencies obtained from the dynamic regression models: (a) Mode $\mathrm{B}_{1}$; (b) Mode $\mathrm{B}_{2}$; (c) Mode $\mathrm{T}_{1}$

The dynamic regression models were subsequently used to predict the natural frequencies after the training period and to detect possible changes in the structural behaviour. The relevant results are presented in terms of prediction errors for modes $\mathrm{B}_{1}, \mathrm{~B}_{2}$ and $\mathrm{T}_{1}$ (Fig. 7) and allow the following comments:

1. before the seismic event, the prediction error is characterized by fluctuations mainly in the field of positive values and does not exhibit sudden changes;

2. a sudden drop is detected corresponding to the earthquake, along with a clear variation of the previous trend. In particular, after 21/06/2013 the prediction error seems to be characterized by a negative offset;

3. during the months following the seismic event, the prediction error neither decreases nor gets back to the original trend. At the opposite, it hovers around the new offset for few weeks and further increases after the summer period.

The previous observations are confirmed by the results obtained using the Shewhart $T$ control chart (where subgroups of 12 hours have been considered) reported in Fig. 8. The inspection of Fig. 8 clearly highlights that:

(a) during the reference period, the $T^{2}$-statistic exhibits limited variations and only few outliers are detected out of the UCL (i.e. the continuous horizontal red line);

(b) just after the earthquake, the $T^{2}$-statistic abruptly exceeds the defined control region and exhibits a large dispersion. Furthermore, as for the prediction error, the variation is not recovered in the months following the seismic event.

(a)

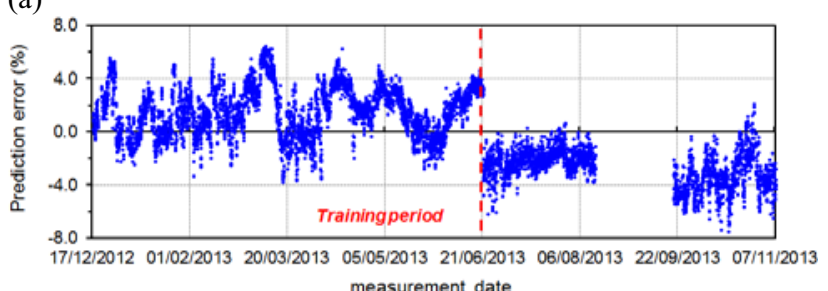

(b)

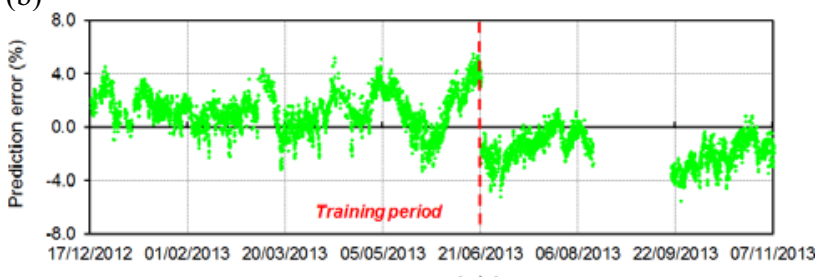
measurement date

(c)

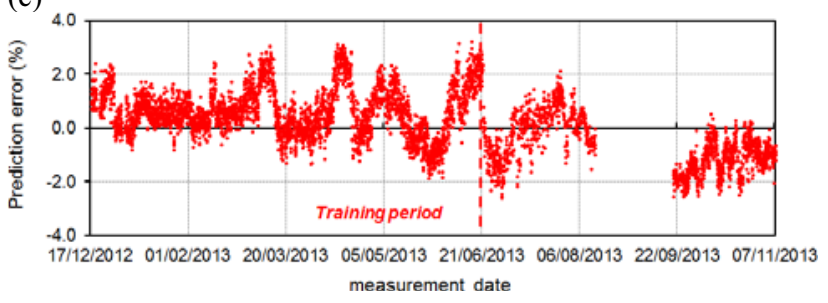

Figure 7. Change in the prediction error induced by the seismic event of 21/06/2013: (a) Mode $\mathrm{B}_{1}$; (b) Mode $\mathrm{B}_{2}$; (c) Mode $\mathrm{T}_{1}$

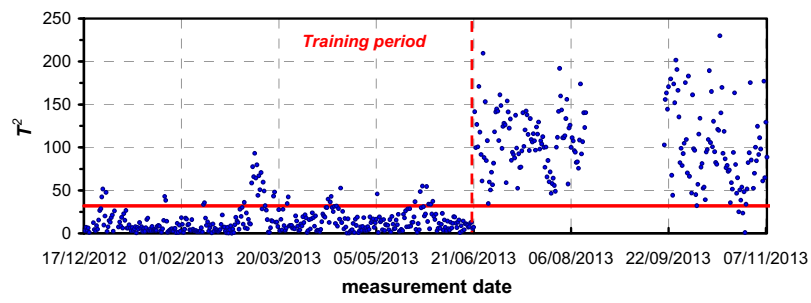

Figure 8. Change in $T^{2}$-statistic induced by the seismic event of $21 / 06 / 2013$

Therefore, both the approaches herein adopted to detect the presence of anomalies, after the removal of the temperature effects, indicate that non-reversible structural changes took place after the Garfagnana earthquake. Furthermore, this conclusion is fully consistent with the variation of the frequency-temperature correlation that was observed both in the short-term period [12] and in the long-term period (Fig. 4). 


\section{Conclusions}

The paper summarizes the main results of the first 15 months of SHM of the Gabbia tower in Mantua, performed through continuous dynamic monitoring.

The adopted methodology is based on the combined use of: (a) a simple monitoring system, consisting of three accelerometers and one temperature sensor installed at the top level of the building; (b) automated modal identification to evaluate the evolution in time of the natural frequencies; (c) dynamic regression models to assess the temperature effects on the natural frequencies, in turn assumed as features sensitive to the structural condition. The SHM strategy herein applied allowed to:

1. accurately track the natural frequencies;

2. evaluate the dependence between frequencies of the global modes and outdoor temperature;

3. distinguish between temperature and damage effects on the natural frequencies of the global modes, revealing the sudden drop of the frequency of the global modes induced by a seismic event;

4. highlight the possible progress of a damage mechanism, involving the upper part of the building, remarked by the significant fluctuations of the natural frequency of the identified local mode.

\section{Acknowledgements}

The investigation was supported by the Mantua Municipality. M. Antico, M. Cucchi (VIBLAB, Laboratory of Vibrations and Dynamic Monitoring of Structures, Politecnico di Milano) and Dr. L. Cantini are gratefully acknowledged for the assistance during the visual inspection, the field tests, the installation and maintenance of the monitoring system.

\section{References}

1. S. Ivorra, F.J. Pallares, Eng. Struct. 25(5): 660-7 (2006)

2. C. Gentile, A. Saisi, Constr. Build. Mater. 21(6): 1311-21 (2007)

3. L.F. Ramos, L. Marques, P.B. Lourenço, G. De Roeck, A. Campos-Costa, J. Roque, Mech. Syst. Signal Pr. 24(5): 1291-305 (2010)

4. C. Gentile, A. Saisi, A. Cabboi, Int. J. Arch. Heritage 9(2): 98-110 (2015)

5. A. Cabboi, Automatic operational modal analysis: challenges and application to historic structures and infrastructures (Ph.D. Thesis, University of Cagliari, 2013)

6. N. Zuccoli, Historic research on the Gabbia tower (Municipality of Mantua internal report, 1988)

7. A. Saisi, C. Gentile, J. Cult. Heritage 16(4): 602-609 (2015)

8. B. Peeters, G. De Roeck, Mech. Syst. Signal Pr. 13(6): 855-78 (1999)

9. B. Peeters, System identification and damage detection in civil engineering (Ph.D. Thesis, Katholieke Universiteit Leuven, 2000)

10. E. Reynders., J. Houbrechts., G. De Roeck, Mech.
Syst. Signal Pr. 29: 228-50 (2012)

11. R. Cantieni, Proc. of the $9^{\text {th }}$ Int. Conf. on Structural Dynamics, EURODYN 2014: 1493-1500 (2014)

12. A. Saisi, C. Gentile, M. Guidobaldi, Constr. Build. Mater. 81: 101-12 (2015)

13. J. Hair, R. Anderson, R. Tatham, W. Black, Multivariate Data Analysis (Prentice Hall, 1998).

14. F. Magalhães, A. Cunha, E. Caetano, Mech. Syst. Signal Pr. 28: 212-228 (2012)

15. E.J. Cross, K.Y. Koo, J.M.W. Brownjohn, K. Worden, Mech. Syst. Signal Pr. 35: 16-34 (2013)

16. L. Ljung, System Identification: Theory for the User (Prentice Hall, New Jersey, 1999)

17. D. Montgomery, Introduction to statistical quality control (Wiley, New York 1997)

18. F. Busatta, Dynamic monitoring and automated modal analysis of large structures: methodologicals aspects and application to a historic iron bridge, (Ph.D. Thesis, Politecnico di Milano, 2012)

19. SVS, http://www.svibs.com/, ARTeMIS Extractor 2011

20. T.O. Kvalseth, Am. Stat. 39: 279-85 (1985) 